\title{
THE SPECTRA OF SUBNORMAL OPERATORS ${ }^{1}$
}

\author{
C. R. PUTNAM
}

ABSTRACT. It is shown that a subnormal operator having its spectrum on a simple closed curve is necessarily normal, and that the corresponding assertion with "subnormal" replaced by "hyponormal" is in general false.

1. All transformations $T$ considered here are bounded operators on a Hilbert space $\mathfrak{S}$. Recall that $T$ is said to be hyponormal if

$$
T^{*} T-T T^{*} \geqq 0,
$$

and subnormal if $T$ has a normal extension $N$ on a Hilbert space $\mathfrak{R} \supset \mathfrak{S}$ such that $N$ leaves $\mathfrak{S}$ invariant and $T=N \mid \mathfrak{S}$. It is known that every subnormal operator is hyponormal but that the converse assertion is false. For properties of subnormal and hyponormal operators, see, e.g., Halmos [5], Putnam [10].

It has recently been shown (Putnam [11]) that if $T$ is hyponormal then its spectrum, $\operatorname{sp}(T)$, satisfies the inequality

$$
\pi\left\|T^{*} T-T T^{*}\right\| \leqq \operatorname{meas}_{2}(\operatorname{sp}(T)),
$$

where meas ${ }_{2}$ denotes ordinary Lebesgue planar measure. In particular, if $T$ is hyponormal and if its spectrum has measure zero, then $T$ is normal. As a special case, if $T$ is hyponormal and if its spectrum is a subset of a simple closed curve having zero planar measure, then $T$ is necessarily normal. It will be shown below, however, that if $T$ is subnormal and if its spectrum is contained in any simple closed curve $C$, thus allowing the possibility that $C$ be an Osgood curve (cf. [8]), then necessarily $T$ is normal. On the other hand, the corresponding assertion becomes false if the hyponormality hypothesis on $T$ is weakened to that of subnormality. Thus, there will be proved the following:

THEOREM. (i) If $T$ is subnormal and if

$$
\operatorname{sp}(T) \subset C,
$$

uhere $C$ is a simple closed curve, then $T$ is normal.

Received by the editors June 8, 1970 .

AMS 1969 subject classifications. Primary 4730, 4740; Secondary 4710,

Key words and phrases. Spectra of hyponormal operators, spectra of subnormal operators, measure of spectrum.

1 This work was supported by a National Science Foundation Research grant. 
(ii) There exists a hyponormal $T$ which is not normal but which satisfies (1.3).

2. Proof of (i). According to the Riemann mapping theorem, the simple closed curve $C$ and its interior, $R$, can be mapped homeomorphically onto $|w| \leqq 1$ by $w=f(z)$, where $f(z)$ is analytic in $R$. In view of Mergelyan's theorem (Mergelyan [7], cf. Rudin [12, p. $386])$, there exists a sequence of polynomials $\left\{p_{n}(z)\right\}, n=1,2, \cdots$, such that, as $n \rightarrow \infty$,

$$
p_{n}(z) \rightarrow f(z) \quad \text { uniformly on } R \cup C .
$$

Let $N$ denote the minimal normal extension on $\mathfrak{\Omega} \mathfrak{S}$ of $T$ with the spectral resolution

$$
N=\int z d K_{z}
$$

Then $N$ and its positive powers, hence all polynomials $p_{n}(N)$, leave $\mathfrak{S}$ invariant. In view of (2.1) (and $\operatorname{sp}(N) \subset \operatorname{sp}(T)$; see Halmos [5, p. 102]),

$$
p_{n}(N)=\int p_{n}(z) d K_{z} \rightrightarrows M \equiv \int f(z) d K_{z}, \quad n \rightarrow \infty,
$$

the convergence being in the norm topology. In particular, the normal operator $M$ also leaves $\mathfrak{W}$ invariant and, as $n \rightarrow \infty$,

$$
p_{n}(T) \rightrightarrows S \equiv M \mid \mathfrak{S} \text {. }
$$

Since $S$ has the normal extension $M, S$ is subnormal. It will next be shown that

$$
\operatorname{sp}(S) \subset\{z:|z|=1\} .
$$

To see this, let $z$ belong to $\operatorname{sp}(S)$ and suppose that $|z| \neq 1$. If $A_{n}$ $=p_{n}(T)$, then $\operatorname{sp}\left(A_{n}\right) \subset p_{n}(C)$, in view of (1.3) and the spectral mapping theorem. It follows from (2.1) that for every $\epsilon>0$ there exists a positive integer $p_{\epsilon}$ such that $\operatorname{sp}\left(A_{n}\right)$ is contained in the annulus $1-\epsilon<|z|<1+\epsilon$ for $n \geqq p_{\epsilon}$. Since $|z| \neq 1$, there exists a number $c>0$ such that $\operatorname{dist}\left(z, \operatorname{sp}\left(A_{n}\right)\right)>c$ for large $n$. Since $A_{n}$ is subnormal, hence hyponormal, it follows that, for large $n,\left\|\left(A_{n}-z I\right) x\right\| \geqq c\|x\|$ and $\left\|\left(A_{n}^{*}-\bar{z} I\right) x\right\| \geqq c\|x\|, x$ in $\mathfrak{S}$. Consequently, by $(2.4),\|(S-z I) x\| \geqq$ $c\|x\|$ and $\left\|\left(S^{*}-\bar{z} I\right) x\right\| \geqq c\|x\|$, so that $z$ does not belong to $\operatorname{sp}(S)$. This proves (2.5).

Since $S$ is hyponormal, it follows from (2.5) that $S$ is normal. (Relation (1.2) can be applied here. However, the result also follows, 
for instance, from earlier work of Donoghue [4] or Stampfli [13, p. 473].) Next, since $M$ is normal (on $\mathfrak{R}$ ) and since $M$ leaves $\mathfrak{S}$ invariant, and the restriction, $S$, of $M$ to $\mathfrak{S}$ is also normal, then $\mathfrak{S}$ reduces $M$; see Berberian [1, p. 160].

If $z=g(w)$ denotes the inverse mapping of $w=f(z)$ then it is clear that

$$
N=\int g(f(z)) d K_{z}
$$

and, by another application of Mergelyan's theorem, that there exist polynomials $q_{n}(w)$ satisfying, as $n \rightarrow \infty$,

$$
q_{n}(w) \rightarrow g(w) \quad \text { uniformly on }|w| \leqq 1 .
$$

Since $\mathfrak{W}$ reduces $M, \mathfrak{W}$ also reduces the operators $q_{n}(M)$. Also, since $q_{n}(M) \rightrightarrows N$ as $n \rightarrow \infty$ (cf. (2.3)), $N$ is reduced by $\mathfrak{S}$, and hence $T=N$, that is, $T$ is normal. This completes the proof of (i).

3. Before giving the proof of (ii) (in $\$ 4$ below), it will be convenient to collect several facts about hyponormal operators. Let $T$ be hyponormal with the rectangular representation

$$
T=H+i J, \quad H \text { and } J \text { selfadjoint. }
$$

Let $H$ have the spectral resolution

$$
H=\int \lambda d E_{\lambda}
$$

It was shown in [9], (see also [10]) that the spectra of $H$ and $J$ are the (real) projections of the spectrum of $T$ onto the real and imaginary axes, respectively. Further, if $\alpha$ denotes a Borel set of the real line and if $T_{\alpha}$ denotes the operator $T_{\alpha}=E(\alpha) T E(\alpha)$ on the Hilbert space $E(\alpha) \mathfrak{E}$, then $T_{\alpha}$ is hyponormal and any reducing subspace on which $T_{\alpha}$ is normal is necessarily also a reducing space of $T$ on which $T$ is normal; see [11, Lemma 5]. Also, it was shown in [11] that

$$
\operatorname{sp}\left(T_{\alpha}\right) \subset \operatorname{sp}(T),
$$

so that $\operatorname{sp}\left(T_{\alpha}\right)$ is a subset of that part of $\operatorname{sp}(T)$ having as its projection on the real axis the closure of $\alpha$. (Actually, (3.3) was proved in [11] when $\alpha$ is an interval but the inclusion is easily generalized to any Borel set.)

An operator $T$ is said to be completely hyponormal if $T$ has no nontrivial reducing subspace on which it is normal. In case $T$ is 
completely hyponormal then, in particular, its real and imaginary parts are absolutely continuous; see [10, p. 42].

4. Proof of (ii). Let $T$ denote any completely hyponormal operator, so that, in particular, $\operatorname{sp}(H)$ has a positive linear measure. Let $\alpha$ be any nowhere dense perfect subset of $\operatorname{sp}(H)$ for which $E(\alpha) \neq 0$, where $H$ has the spectral resolution (3.2). (Consequently, $\alpha$ is a Cantor set of positive linear measure.) Let $T_{\alpha}$ have the rectangular decomposition

$$
T_{\alpha}=H_{\alpha}+i J_{\alpha},
$$

where $H_{\alpha}=\int_{\alpha} \lambda d E_{\lambda}$ and $J_{\alpha}=E(\alpha) J E(\alpha)$ has the spectral resolution

$$
J_{\alpha}=\int \lambda d F_{\lambda}^{(\alpha)}
$$

Then $\operatorname{sp}\left(H_{\alpha}\right)$ is the projection of $\operatorname{sp}\left(T_{\alpha}\right)$ onto the real axis. Further (cf. §3), $T_{\alpha}$ is completely hyponormal and (3.3) holds. Let $\beta$ denote any nowhere dense perfect subset of $\operatorname{sp}\left(J_{\alpha}\right)$ for which $F^{(\alpha)}(\beta) \neq 0$ and define $T_{\alpha \beta}$ as

$$
T_{\alpha \beta}=F^{(\alpha)}(\beta) T_{\alpha} F^{(\alpha)}(\beta) .
$$

Then, as in the discussion above, $T_{\alpha \beta}$ is completely hyponormal and

$$
\operatorname{sp}\left(T_{\alpha \beta}\right) \subset \alpha \times \beta .
$$

In order to complete the proof of (ii) it is sufficient to show that there exists a simple closed curve $C$ which contains the product set $\alpha \times \beta$. But the existence and, in fact, explicit construction of such a curve can be established in a manner similar to that of the standard construction of space-filling (Peano) curves; see, for instance, Hobson [6, pp. 451-458]. (The author is indebted to R. B. Darst for pointing out the existence of such a curve $C$ and also for calling his attention to the paper of Darst and Goffman [3].)

5. Remarks. It has been noted by K. Clancey [2, p. 22], as well as by J. G. Stampfli, that if $T$ is subnormal and if its spectrum is nowhere dense and does not separate the plane, then $T$ must be normal. The example of $\$ 4$ above shows that the corresponding assertion can be false if "subnormal" is replaced by "hyponormal," and, moreover, that there exists a completely hyponormal operator having a totally disconnected spectrum.

The author is grateful to the referee for furnishing the following alternate proof of part (i) of the Theorem and which is due to J. G. 
Stampfli. Since $T$ is subnormal then $\operatorname{sp}(T)$ is a spectral set of $T$. If $f(z)$ is defined as in $\$ 2$ above then, in view of (2.1), the image of $\operatorname{sp}(T)$ under $f(z)$ is a spectral set of $f(T)$; see von Neumann [14, p. 266]. Consequently, the operator $f(T)$, hence also $T=g(f(T))$, is normal.

\section{REFERENCES}

1. S. K. Berberian, Introduction to Hilbert space, University Texts in Math. Sci., Oxford Univ. Press, New York, 1961. MR 25 \#1424.

2. K. Clancey, Spectral properties of semi-normal operators, Thesis, Purdue University, Lafayette, Ind., 1969.

3. R. Darst and C. Goffman, A Borel set which contains no rectangles, Amer. Math. Monthly 77 (1970), 728-731.

4. W. F. Donoghue, On a problem of Nieminen, Inst. Hautes Études Sci. Publ. Math. No. 16 (1963), 31-33. MR 27 \#2864.

5. P. R. Halmos, A Hilbert space problem book, Van Nostrand, Princeton, N.J., 1967. MR $34 \# 8178$.

6. E. W. Hobson, The Theory of functions of a real variable. Vol. 1, 2nd ed., Cambridge Univ. Press, New York, 1927.

7. S. N. Mergeljan, Uniform approximations to functions of a complex variable, Uspehi Mat. Nauk 5 (1952), no. 2 (48), 31-122; English transl., Amer. Math. Soc. Transl. (1) 3 (1962), 294-391. MR 14, 547; MR 15, 612.

8. W. F. Osgood, A Jordan curve of positive area, Trans. Amer. Math. Soc. 4 (1903), $107-112$.

9. C. R. Putnam, On the spectra of semi-normal operators, Trans. Amer. Math. Soc. 119 (1965), 509-523. MR 32 \#2913.

10. - Commutation properties of Hilbert space operators and related topics, Ergebnisse der Mathematik und ihrer Grenzgebiete, Band 36, Springer-Verlag, New York, 1967. MR 36 \#707.

11. - - An inequality for the area of hyponormal spectra, Math. Z. 116 (1970), 323-330.

12. W. Rudin, Real and complex analysis, McGraw-Hill, New York, 1966. MR 35 $\# 1420$.

13. J. G. Stampfli, Hyponormal operators and spectral density, Trans. Amer. Math. Soc. 117 (1965), 469-476. MR 30 \#3375.

14. J. von Neumann, Eine Spektraltheorie für allgemeine Operatoren eines unitären Raumes, Math. Nachr. 4 (1951), 258-281. MR 13, 254.

Purdue University, LafayetTe, Indiana 47907 